\title{
PMMA-augmentation of incompletely cannulated pedicle screws: A cadaver study to determine the benefits in the osteoporotic spine
}

\author{
H. Goost ${ }^{\mathrm{a}, 1, *}$, C. Deborre ${ }^{\mathrm{a}, 1}$, D.C. Wirtz ${ }^{\mathrm{a}}$, C. Burger ${ }^{\mathrm{a}}$, A. Prescher ${ }^{\mathrm{b}}$, C. Fölsch ${ }^{\mathrm{c}}$, \\ R. Pflugmacher ${ }^{\mathrm{a}}$ and K. Kabir ${ }^{\mathrm{a}}$ \\ ${ }^{a}$ Department of Orthopaedics and Trauma Surgery, University Hospital Bonn, Bonn, Germany \\ b Institute of Anatomy, University Hospital RWTH-Aachen, Aachen, Germany \\ ${ }^{\mathrm{c}}$ Department of Orthopaedics, University Hospital Marburg, Marburg, Germany
}

Received 3 March 2014

Accepted 25 March 2014

\begin{abstract}
.
BACKGROUND: Pedicle screw pullout due to poor bone quality, mainly caused by osteoporosis, is a common problem in spine surgery. Special implants and techniques, especially PMMA augmentation, were developed to improve the fixation of pedicle screws. PMMA injection into a pilot hole or through a screw involves the same risks as vertebroplasty or kyphoplasty, regardless of the technique used. Especially when using fully cannulated screws anterior leakage is possible.

OBJECTIVE: To prove PMMA injection is safe and possible without leakage through an incompletely cannulated screw and also increases pullout forces in the osteoporotic vertebra.

METHODS: Incompletely cannulated pedicle screws were tested by axial pullout in human cadavers, divided into osteoporotic and non-osteoporotic groups. Non-augmented and PMMA-augmented pedicle screws were compared. Twenty-five human vertebrae were measured by DEXA and divided into osteoporotic and non-osteoporotic groups. In each vertebra both pedicles were instrumented with the new screw (WSI-Expertise $6 \times 45 \mathrm{~mm}$, Peter Brehm Inc. Germany); the right screw was augmented with a $3 \mathrm{~mL}$ PMMA injection through the screw. On each screw axial pullout was performed after X-ray and CT scan.

RESULTS: Radiographs and CT scans excluded PMMA leakage. Cement was distributed in the middle and posterior third of the vertebrae. Pullout forces were significantly higher after pedicle screw augmentation, especially in the osteoporotic bone. All augmented pedicle screws showed higher pullout forces compared with the unaugmented screws.

CONCLUSIONS: We minimized the risk of leakage by using a screw with a closed tip. On the whole, PMMA augmentation through an incompletely cannulated pedicle screw is safe and increases pullout forces in osteoporotic bone to the level of healthy bone. Therefore the new incompletely cannulated screw can be used for pedicle screw augmentation.
\end{abstract}

Keywords: Pedicle screw fixation, PMMA, osteoporosis, pullout test, spine biomechanics, spine degeneration, spine fracture, screw loosening

\footnotetext{
${ }^{1}$ Both authors contributed equally to this paper.

${ }^{*}$ Corresponding author: Hans Goost, Department of Orthopedic and Trauma Surgery, University Hospital Bonn, SigmundFreud-Str. 25, D 53125 Bonn, Germany. E-mail: hans.goost@ukb.uni-bonn.de.
}

0928-7329/14/\$27.50 (C) 2014 - IOS Press and the authors. All rights reserved

This article is published online with Open Access and distributed under the terms of the Creative Commons Attribution NonCommercial License. 


\section{Introduction}

Poor bone quality remains a major challenge to the spine surgeon. Osteoporosis is a major cause of this, and its prevalence and incidence will increase due to an aging population in the coming years [1].

A major sequela of osteoporosis is a vertebral compression fracture. A large number of vertebral compression fractures can be treated non-operatively, or by vertebroplasty or kyphoplasty. Unstable burst fractures with a relevant posterior wall fragment, progressive collapse of the vertebral body, and neurologic complications require instrumentation [2]. Also a growing number of surgical procedures in degenerative spine diseases such as instability, spinal stenosis, and osteochondrosis require stabilization in the osteoporotic spine [3].

Pedicle screw pullout or creeping loosening is a frequent complication in the osteoporotic spine. The bone between the screw threads fractures faster when bone quality and quantity is decreased. Concepts to improve pedicle screw pullout therefore include changes in pedicle screw design, implantation technique, and augmentation [4].

PMMA augmentation of a pedicle screw is a reliable method to improve fixation in the osteoporotic spine [5]. Actual augmentation techniques involve special completely cannulated pedicle screws with one anterior hole and two to six lateral holes. With an open tip there is a risk of PMMA leakage with bicortical fixation. The incompletely cannulated pedicle screw was developed to address these problems.

In this study we tested the biomechanical properties of this new self-tapping pedicle screw with incomplete cannulation and two lateral holes. Our hypothesis was that the new screw design would increase the pullout forces compared with a non-cemented screw. Furthermore the PMMA depot should be placed in a biomechanically advantageous position in the posterior half of the vertebral body to prevent cement leakage, especially anteriorly [6].

\section{Methods}

\subsection{Ethics}

Written informed consent from the body donors was obtained by last will and testament according to the requirements of the ethics committee at the Technical University Aachen. According to the local ethics committee at the University Hospital Bonn, a separate approval of the study and an informed consent for use of cadaveric samples was not necessary, as these bodies had been donated to medical scientific studies, including dissection and biomechanical studies, by the deceased.

\subsection{Vertebral bodies}

Two thoracic vertebrae (T-12) and 23 lumbar vertebrae (L1-L5) were harvested from 12 human cadavers. Those specimens that showed apparent bone lesions or bone metabolic abnormalities on plain radiograms were excluded. Adherent soft tissues such as muscles, ligaments, and intervertebral discs were removed and each vertebral body was separated carefully. Bone mineral density (BMD) was measured using the dual-energy radiograph absorptiometry (DEXA) method (Siemens QDR 2000, Erlangen, Germany). Osteoporosis was defined as a BMD (bone mass density) lower $0.8 \mathrm{~g} / \mathrm{cm}^{3}$. Then the vertebrae were divided into an osteoporotic and a non-osteoporotic group. Prior to the mechanical tests, specimens were kept hydrated in plastic bags. During the tests, the room temperature was kept at $293^{\circ} \mathrm{K}$ $\left(20^{\circ} \mathrm{C}, 68^{\circ} \mathrm{F}\right)$ and specimens were prevented from drying by applying a covering of resin. 


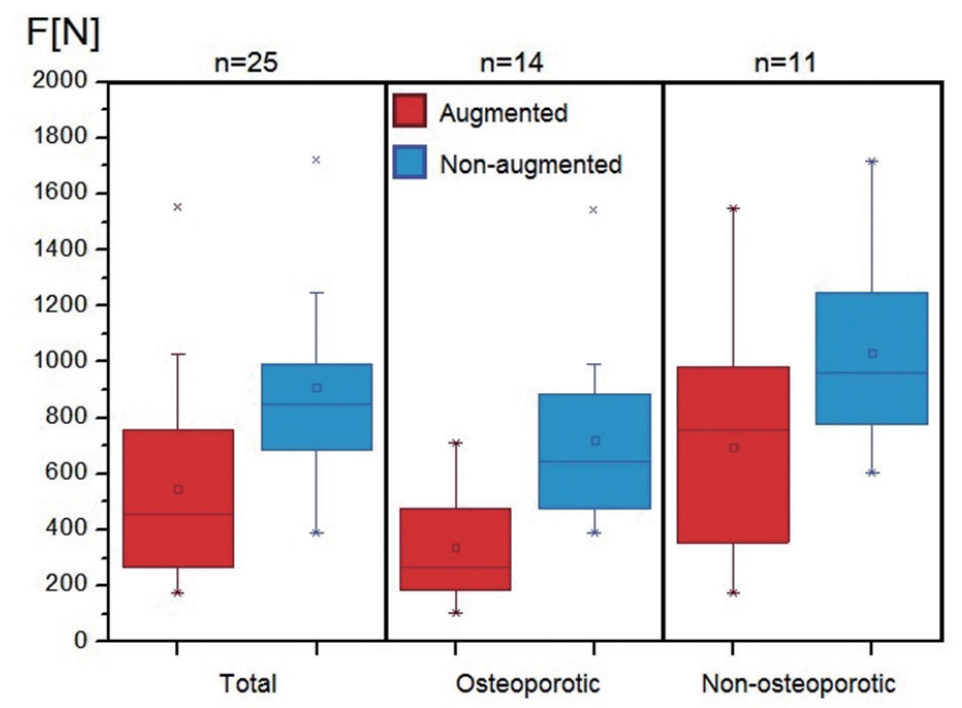

Fig. 1. Pullout forces. (Colours are visible in the online version of the article; http://dx.doi.org/10.3233/THC-140815)

\subsection{Screw}

For the tests we used a newly developed titanium polyaxial pedicle screw (WSI-Expertise $6 \times 45 \mathrm{~mm}$, Peter Brehm Inc. Germany). The self-tapping screw was partially cannulated. The tip was closed. Two lateral holes were located in the third and fourth thread windings, $1 \mathrm{~cm}$ above the tip (Fig. 1). A peak adapter was used to facilitate PMMA injection. Screws $45 \mathrm{~mm}$ in length and $6 \mathrm{~mm}$ in diameter were chosen to ensure a standardized experimental setup.

\subsection{Implantation technique}

Under fluoroscopic guidance the cortex over the pedicle was perforated using an awl. The pedicle screws were implanted into both pedicles. Through the cementing adapter $3 \mathrm{~mL}$ of PMMA (Spineplex, Stryker, Warzaw, USA) was injected into the right screw. The distribution of the PMMA around the screws was monitored under fluoroscopy. The left pedicle screw remained unaugmented as control. After PMMA injection axial and lateral radiographs were obtained to document cement leakage and distribution in the vertebra.

\subsection{Cement distribution}

To validate the cement distribution in the vertebral body we developed a scheme with three zones on the craniocaudal radiograph. Every vertebral body was divided from the anterior cortex to the posterior wall into three zones Zone one represented the anterior third, zone two the middle third, and zone three the posterior third at the posterior wall. The cement depot should be located in zones two and three or in zone three only.

\subsection{Mechanical testing}

The prepared vertebrae were kept overnight at room temperature to guarantee PMMA settlement. The next day the vertebrae were immersed up to the posterior wall in PMMA (Technovit 4004, Hereaus- 


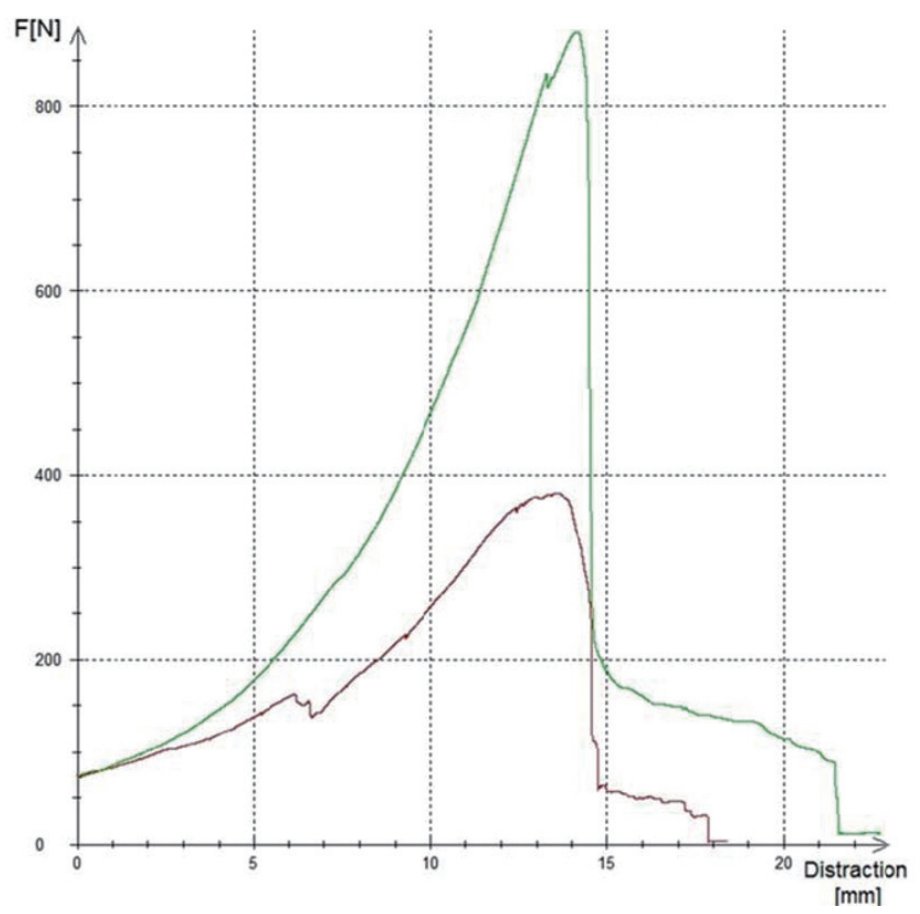

Fig. 2. Pedicle screw loosing in experimental setup. (Colours are visible in the online version of the article; http://dx.doi. org/10.3233/THC-140815)

Kulzer, Germany). The resulting PMMA-vertebra block was mounted on a testing device (Zwick/Roell Zmartpro, Germany). Two steel wires were drawn through the spinal canal and over the block; the pedicle screw was connected to the implantation instrument and also connected with a wire. To keep the distance and prevent the bone material from being sawed by the steel wire, we fed the wire through a short piece of flexible tube. Both wires were connected to the pullout ram and adjusted axially (Fig. 2).

Pullout testing was begun with the non-augmented screw, followed by the augmented screw. As starting force we chose $75 \mathrm{~N}$, an approach speed of $20 \mathrm{~mm} / \mathrm{min}$, and a testing speed of $2 \mathrm{~mm} / \mathrm{min}$. The test was stopped automatically after a complete pullout or loss of force lower than 25 percent of the maximum (Figs 3 and 4). For control and evaluation purposes we used the analysis software "testXpert" (Zwick/Roell).

\subsection{Statistical analysis}

The mean maximum pullout strength was compared between the 2 screws by paired Student's t-tests. The relationship between the maximum pullout strength and BMD was analyzed by Pearson correlation coefficient test. $P<0.01$ was considered significant. SPSS 11.0 (SPSS Inc., Chicago, Il) was used for statistical analysis.

\section{Results}

The Spineplex cement was easily injected through the peak cementing adapter. Leakage of PMMA, especially anteriorly or into the spinal canal, was not observed. 


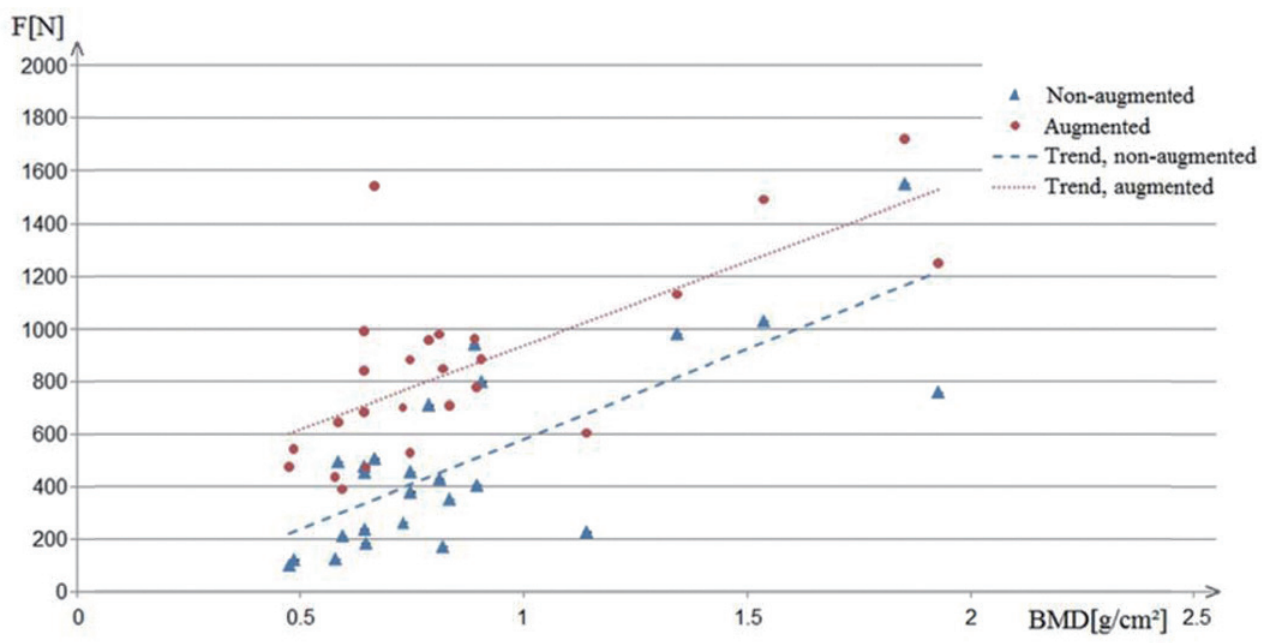

Fig. 3. Trend curve - pullout forces vs. BMD. (Colours are visible in the online version of the article; http://dx.doi.org/10.3233/ THC-140815)

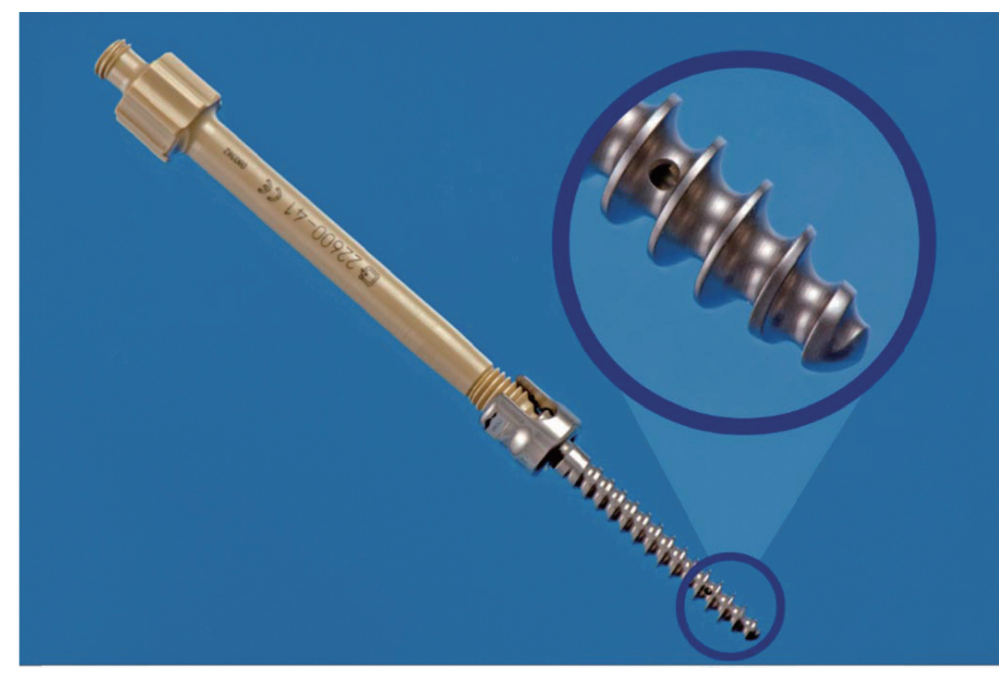

Fig. 4. Incompletly cannulated pedicle screw with cementing adapter. (Colours are visible in the online version of the article; http://dx.doi.org/10.3233/THC-140815)

The mean BMD of all specimens was $0.877 \mathrm{~g} / \mathrm{cm}^{3}$ ( $\min / \max 0.476 / 1.927 ; \mathrm{SD} \pm 0.332$ ). Due to the definition of osteoporosis 14 specimens had a BMD lower than $0.8 \mathrm{~g} / \mathrm{cm}^{3}$ (mean 0.641; min/max $0.476 / 0.788 ; \mathrm{SD} \pm 0.093$ ). The non-osteoporotic group consisted of 11 specimens with a mean BMD of $1.178 \mathrm{~g} / \mathrm{cm}^{3}(\mathrm{~min} / \max 0.812 / 1.927 ; \mathrm{SD} \pm 0.423)$.

The radiographic images of all vertebrae excluded any PMMA leakage. The cement distribution in the three zones showed cement only in zones 2 and 3 (Fig. 4). Cement was not observed in the anterior part of any vertebra.

Overall we observed an increase in the mean axial pullout strength of $173 \%$ when using cement augmented screws (non-cemented $496 \mathrm{~N}$ vs. cemented $858 \mathrm{~N}, p \leqslant 0.001$ ). In the osteoporotic group the mean pullout force of the non-cemented screws was $339 \mathrm{~N}$ vs. $721 \mathrm{~N}$ for the cemented screws $(p \leqslant$ 


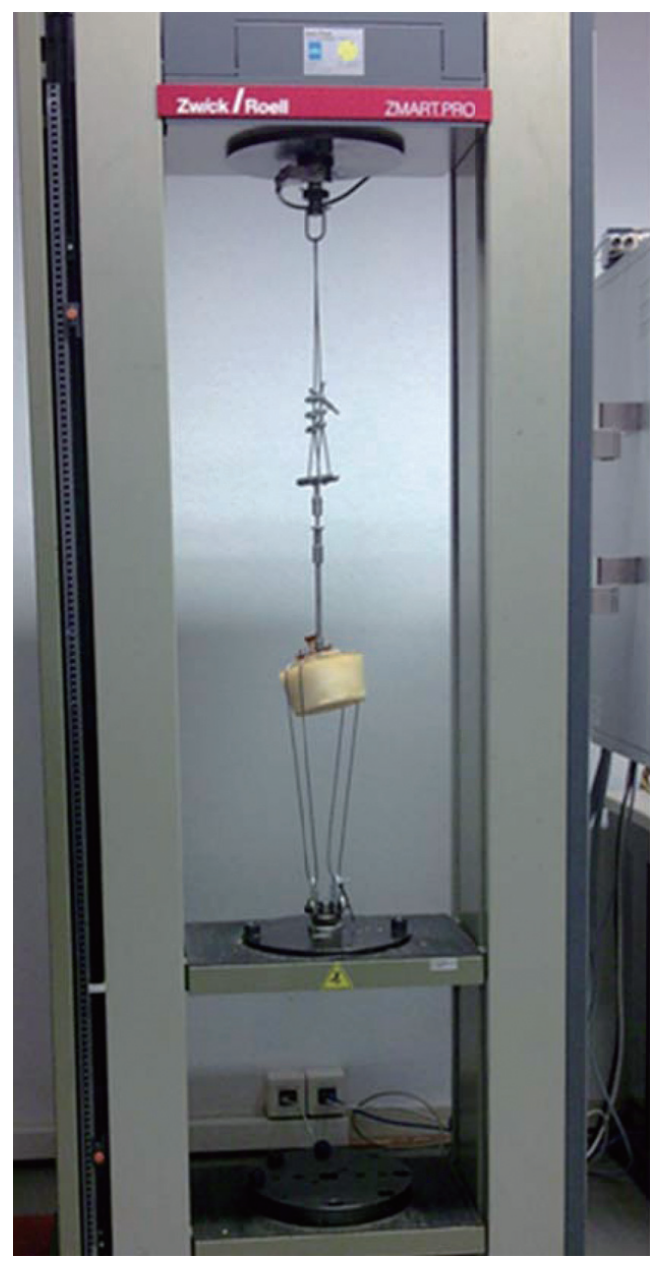

Fig. 5. Axial pullout setup with Zwick-Roell Zmart Pro. (Colours are visible in the online version of the article; http://dx.doi. org/10.3233/THC-140815)

0.001). Similarly, the pullout force increased in the non-osteoporotic group from $696 \mathrm{~N}$ for the noncemented screws or $1066 \mathrm{~N}$ for the cemented screws $(p \leqslant 0.001$; Fig. 5). A typical load-time curve in our experimental setup is displayed in Fig. 6. A correlation between BMD and pullout forces in nonaugmented and augmented screws was observed but was not statistically significant (Fig. 7).

\section{Discussion}

Successful surgery in the elderly spine requires special pedicle screws and implantation techniques adapted to osteoporosis. In our trial we tested cement augmentation through a new incompletely cannulated pedicle screw in osteoporotic and non-osteoporotic fresh frozen cadaver vertebrae. Incompletely cannulated pedicle screws were chosen to avoid anterior cement leakage. A further goal was to place the PMMA depot close to the posterior wall.

Our study showed significantly higher pullout forces for the incompletely cannulated PMMAaugmented pedicle screws, especially in the osteoporotic bone. The mean pullout force for the osteo- 


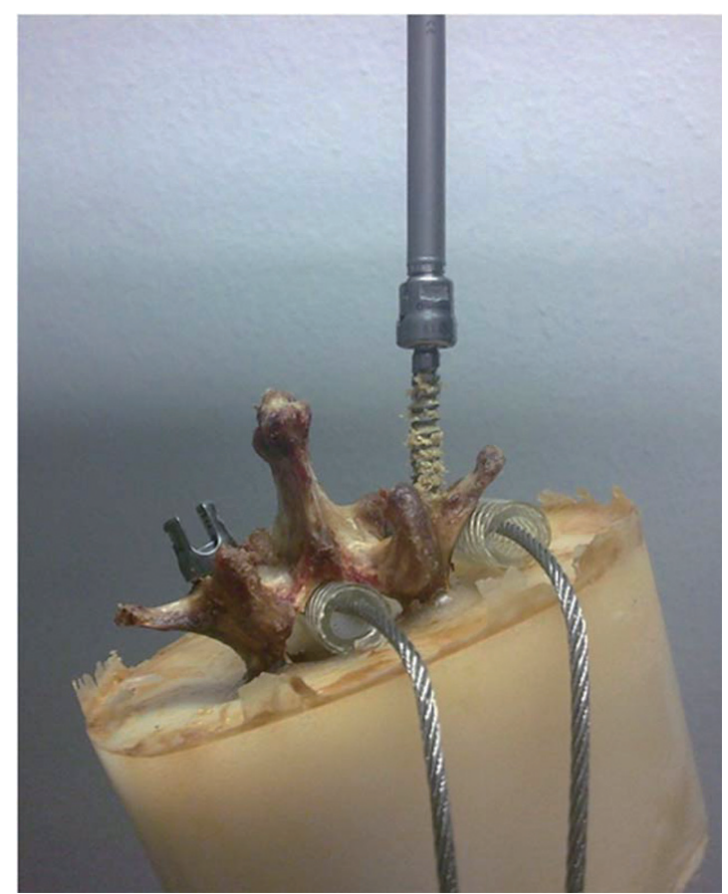

Fig. 6. Pedicle screw loosing in experimental setup. (Colours are visible in the online version of the article; http://dx.doi.org/10.3233/THC-140815)

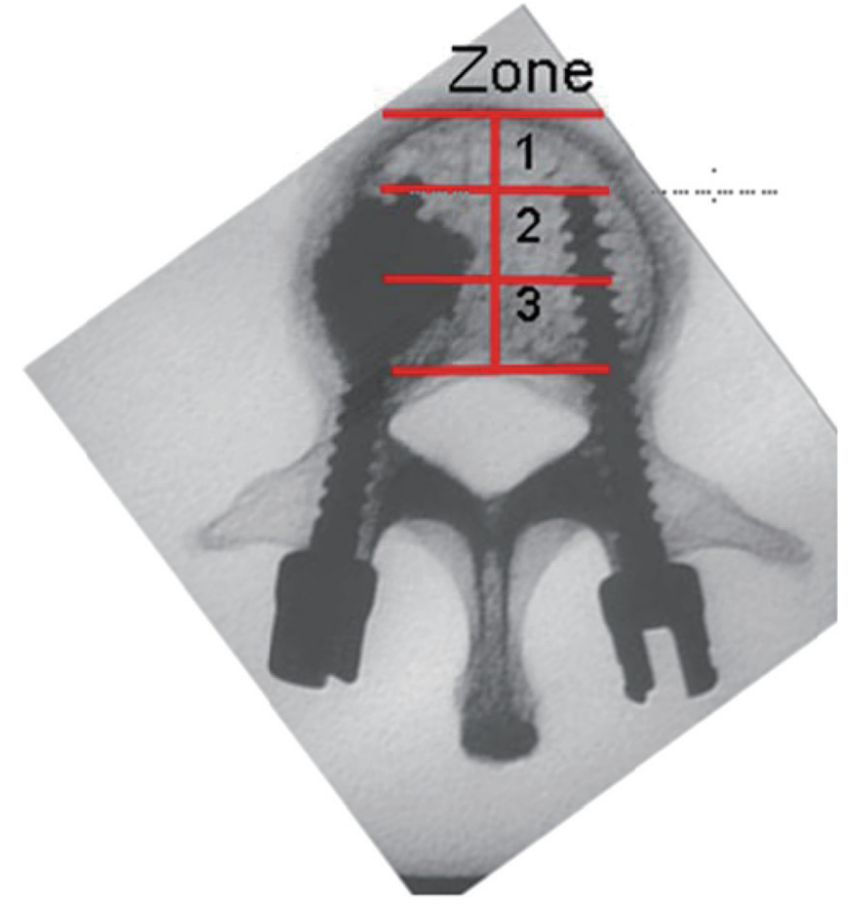

Fig. 7. Cement allocation zones. (Colours are visible in the online version of the article; http://dx.doi.org/10.3233/THC-140815)

porotic vertebrae was $339 \mathrm{~N}$ for the non-augmented screws compared with $721 \mathrm{~N}$ for the cemented screws. Furthermore, no cement leakage was observed. Through the lateral screw holes the cement creates a depot close to the posterior wall in a more favorable biomechanical position.

Pedicle screws are the crucial interface in spine surgery. Every change in design or implantation technique affects the pullout forces of the screw. In revision surgery or severe osteoporosis pedicle screws require augmentation. Special cannulated screws with varying numbers of anterior or lateral holes in different positions are available for augmentation. Common pedicle screws can be augmented by filling the tapped holes with PMMA [7]. It seems that conical screws with lateral perforations show better results than screws with an anterior hole [8].

Chen showed that the arrangement of holes and the position of the cement depot are very important factors in increasing pedicle screw pullout in cut bone [6]. A cement depot at the tip or middle of the screw is inferior to a depot in the posterior part. For clinical use he suggests a cement depot close to the posterior wall of the vertebra. Pfeiffer also demonstrated that the best position for a PMMA depot is close to the posterior wall. Until now the focus of interest has been on the biomechanical effects of injecting PMMA through the screw; cement application and the position of the cement depot have not been of interest. Therefore we redesigned a common cannulated polyaxial pedicle screw so that the tip is closed and the cement is pressed out through two lateral holes one centimetre from the screw head.

In addition to pedicle screw design, the technique of augmentation and the cement itself are important. PMMA augmentation seems to be the technique most frequently used in routine clinical practice [9]. The required volume of PMMA is thought to be $2-3 \mathrm{~mL}$; a higher volume of PMMA does not guarantee higher pullout forces [10]. Bone grafts have been shown to be inferior to cement augmentation. Calcium phosphate cement augmentation shows a significant increase in pullout forces [11]. In contrast to 
PMMA, calcium phosphate cements are biocompatible and degradable, but need up to 24 hours to set. Therefore this material is not acceptable for routine clinical use.

However, PMMA augmentation also involves risks of fatal complications. Weinstein and Schulze independently showed that anterior cortex violation occurs in up to $20 \%$ of all pedicle screw instrumentations $[12,13]$. Leakage due to improper pedicle screw placement can damage tissue when the cement heats up in the exothermic reaction. Low-viscosity cement is sometimes pressed out of the vertebral body and washed away. This leakage can cause pulmonary embolism [14].

In contrast to the above mentioned PMMA augmentation through a cannulated screw, augmentation can also performed using different techniques. In routine clinical practice, PMMA is frequently injected into a predrilled hole. Some authors prefer a kyphoplasty first and then place the pedicle screw into this cement depot. The choice of pedicle screw augmentation technique depends on possible complications and the spine surgeon's preferences and experience.

Our test configuration does not reflect the physiologic burden on an implanted pedicle screw. Cyclic loading fatigue tests are recommended by some authors to simulate the longitudinal forces pedicle screws must withstand. Burval found a decrease of $30 \%$ in axial pullout force after loading tests [15]. We feel that cyclic loading tests may be dispensed with for our line of inquiry because they will only show a decrease in pullout forces and not a systematic failure in the whole experimental setup. Although most authors tested their augmented pedicle screws in an axial pullout model as has been done here, others suggest initial swing testing and followed by a defined number of cycles testing axial pullout.

Pedicle screw augmentation is a common technique in routine clinical practice. Chang showed in a retrospective study with 41 patients and 300 screws that cement augmentation is a safe and reliable technique [16]. Frankel retrospectively monitored 25 patients with bone softening due to osteoporosis or tumor and used pilot-hole filling for augmentation [17]. He reports excellent results without screw loosening or pullout. No single technique has yet clearly demonstrated superiority in laboratory or clinical studies [18].

Pedicle screw augmentation with PMMA significantly increases pullout forces in the human cadaver model. The benefit of augmentation is seen especially in osteoporotic vertebrae. Injection through a screw is safe and avoids anterior cement leakage. Due to their incomplete cannulation these new pedicle screws can be used in both sacral and bicortical lumbar instrumentation. Clinical studies are needed to demonstrate the efficacy of the concept in routine clinical practice.

\section{References}

[1] Burge R, Dawson-Hughes B, Solomon DH, Wong JB, King A, Tosteson A. Incidence and economic burden of osteoporosis-related fractures in the United States, 2005-2025. J Bone Miner Res. 2007; 22(3): 465-75.

[2] Kim DH, Vaccaro AR. Osteoporotic compression fractures of the spine; current options and considerations for treatment. Spine J. 2006; 6(5): 479-87.

[3] Delank KS, Rollinghoff M, Eysel-Gosepath K, Sobottke R, Eysel P. [Degeneration and osteoporosis of the spine. Is there a modified procedure?]. Orthopade. 2010; 39(4): 425-31.

[4] Wittenberg RH, Lee KS, Shea M, White AA, 3rd, Hayes WC. Effect of screw diameter, insertion technique, and bone cement augmentation of pedicular screw fixation strength. Clin Orthop Relat Res. 1993(296): 278-87.

[5] Moon BJ, Cho BY, Choi EY, Zhang HY. Polymethylmethacrylate-augmented screw fixation for stabilization of the osteoporotic spine: A three-year follow-up of 37 patients. J Korean Neurosurg Soc. 2009; 46(4): 305-11.

[6] Chen LH, Tai CL, Lai PL, Lee DM, Tsai TT, Fu TS, et al. Pullout strength for cannulated pedicle screws with bone cement augmentation in severely osteoporotic bone: influences of radial hole and pilot hole tapping. Clin Biomech (Bristol, Avon). 2009; 24(8): 613-8.

[7] Chao KH, Lai YS, Chen WC, Chang CM, McClean CJ, Fan CY, et al. Biomechanical analysis of different types of pedicle screw augmentation: A cadaveric and synthetic bone sample study of instrumented vertebral specimens. Med Eng Phys. 2013; 35(10): 1506-12. 
[8] Kafchitsas K, Geiger F, Rauschmann M, Schmidt S. [Cement distribution in vertebroplasty pedicle screws with different designs]. Orthopade. 2010; 39(7): 679-86.

[9] Goost H, Kabir K, Wirtz DC, Deborre C, Karius T, Pflugmacher R, et al. [PMMA augmentation of pedicle screws: Results of a survey in Germany]. Z Orthop Unfall. 2012; 150(3): 318-23.

[10] Pare PE, Chappuis JL, Rampersaud R, Agarwala AO, Perra JH, Erkan S, et al. Biomechanical evaluation of a novel fenestrated pedicle screw augmented with bone cement in osteoporotic spines. Spine (Phila Pa 1976). 2011; 36(18): E1210-4.

[11] Renner SM, Lim TH, Kim WJ, Katolik L, An HS, Andersson GB. Augmentation of pedicle screw fixation strength using an injectable calcium phosphate cement as a function of injection timing and method. Spine (Phila Pa 1976). 2004; 29(11): E212-6.

[12] Weinstein JN, Spratt KF, Spengler D, Brick C, Reid S. Spinal pedicle fixation: reliability and validity of roentgenogrambased assessment and surgical factors on successful screw placement. Spine (Phila Pa 1976). 1988; 13(9): 1012-8.

[13] Schulze CJ, Munzinger E, Weber U. Clinical relevance of accuracy of pedicle screw placement. A computed tomographic-supported analysis. Spine (Phila Pa 1976). 1998; 23(20): 2215-20; discussion 20-1.

[14] Rollinghoff M, Siewe J, Eysel P, Delank KS. Pulmonary cement embolism after augmentation of pedicle screws with bone cement. Acta Orthop Belg. 76(2): 269-73.

[15] Burval DJ, McLain RF, Milks R, Inceoglu S. Primary pedicle screw augmentation in osteoporotic lumbar vertebrae: biomechanical analysis of pedicle fixation strength. Spine (Phila Pa 1976). 2007; 32(10): 1077-83.

[16] Chang MC, Liu CL, Chen TH. Polymethylmethacrylate augmentation of pedicle screw for osteoporotic spinal surgery: A novel technique. Spine (Phila Pa 1976). 2008; 33(10): E317-24.

[17] Frankel BM, Jones T, Wang C. Segmental polymethylmethacrylate-augmented pedicle screw fixation in patients with bone softening caused by osteoporosis and metastatic tumor involvement: A clinical evaluation. Neurosurgery. 2007; 61(3): 531-7; discussion 7-8

[18] Chen LH, Tai CL, Lee DM, Lai PL, Lee YC, Niu CC, et al. Pullout strength of pedicle screws with cement augmentation in severe osteoporosis: A comparative study between cannulated screws with cement injection and solid screws with cement pre-filling. BMC Musculoskelet Disord. 2011; 12: 33. 